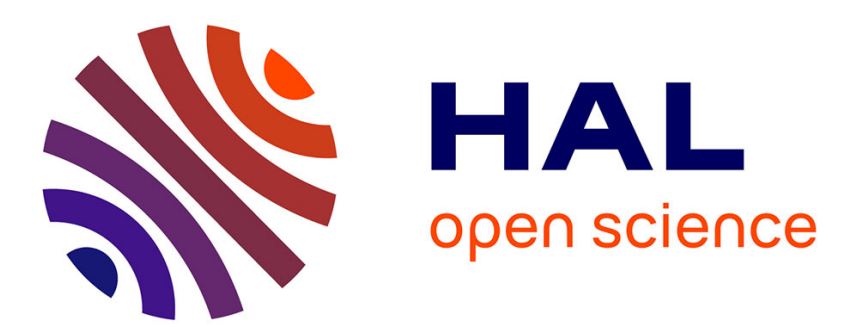

\title{
Acyl Transfer Strategies as Transient Activations for Enantioselective Synthesis
}

\author{
Jean Rodriguez, Adrien Quintard
}

\section{To cite this version:}

Jean Rodriguez, Adrien Quintard. Acyl Transfer Strategies as Transient Activations for Enantioselective Synthesis. Synthesis: Journal of Synthetic Organic Chemistry, 2019, 51 (09), pp.1923-1934. 10.1055/s-0037-1611743 . hal-02279701

\section{HAL Id: hal-02279701 \\ https://hal.science/hal-02279701}

Submitted on 5 Sep 2019

HAL is a multi-disciplinary open access archive for the deposit and dissemination of scientific research documents, whether they are published or not. The documents may come from teaching and research institutions in France or abroad, or from public or private research centers.
L'archive ouverte pluridisciplinaire HAL, est destinée au dépôt et à la diffusion de documents scientifiques de niveau recherche, publiés ou non, émanant des établissements d'enseignement et de recherche français ou étrangers, des laboratoires publics ou privés. 


\section{Acyl Transfer Strategies as Transient Activations for Enantioselective Synthesis}

Jean Rodriguez

Adrien Quintard* (D)

Aix Marseille Univ, CNRS, Centrale Marseille, iSm2, Marseille,

France

adrien.quintard@univ-amu.fr

Dedicated to Michel Quintard on the occasion of his retirement
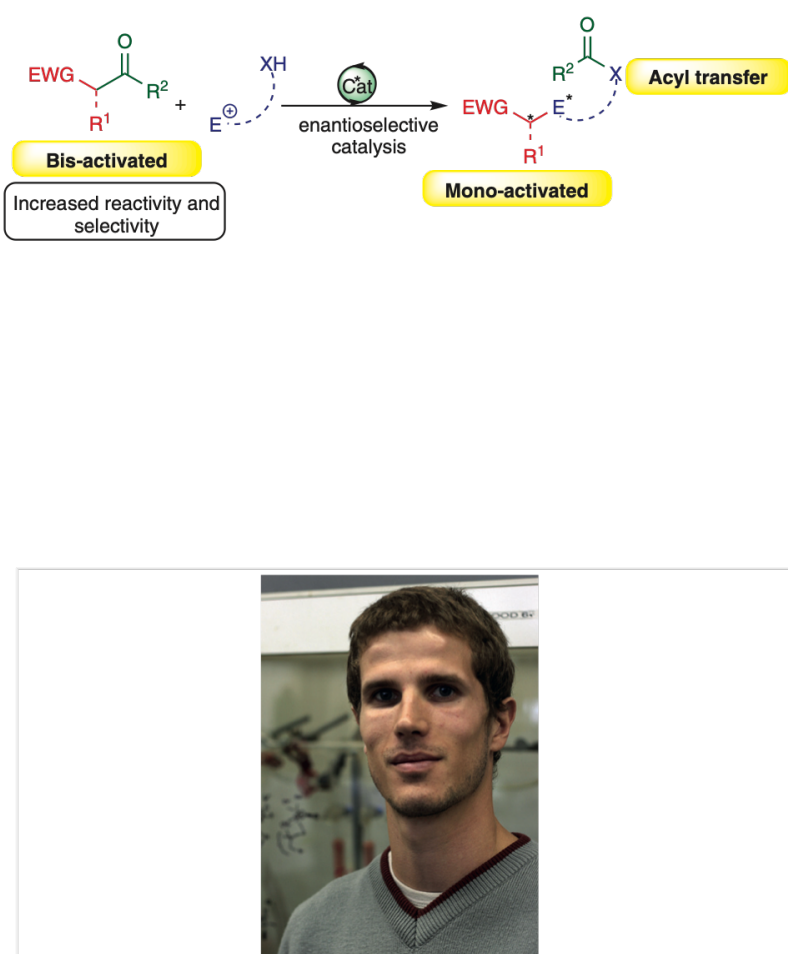

Adrien Quintard studied chemistry at the University of Toulouse (IUT) and then at the CPE University of Lyon. He completed his Ph.D. in 2011 under the supervision of Prof. A. Alexakis at the University of Geneva. He subsequently moved to the University of Stanford for a first postdoctoral stay working with Prof. B. M. Trost. After moving to Aix-Marseille University in 2012 for a second postdoctoral position, he obtained an ANR research grant in 2013 allowing him to start his own research program on the development of multi-catalyzed transformations before becoming a CNRS researcher in 2014. His research interests encompass a broad range of areas of organic chemistry including organocatalysis, metal catalysis, their combination in multi-catalysis and the implementation of these methods in multi-step synthesis with more recent applications in supramolecular chemistry and materials. Recently, his contributions were recognized by the award of the young researcher 'Emergence' prize from the Organic Division of the French Chemical Society and he is also a recipient of a Thieme Chemistry Journals Award.

many different functional groups are insufficiently reactive to be engaged in a direct transformation requiring additional steps and reagents for their activation.

For example, nucleophilic functionalization $\alpha$ - to an electron-withdrawing group often involves the generation of enolates that are sometimes not compatible with an enantioselective transformation (Scheme 1a). This can be due to the difficulty in generating the desired enolate, often requiring strong bases or harsh conditions, resulting in additional undesired waste and a lack of control during the 
nucleophilic addition. In the case of aliphatic ketones, an additional issue of regiocontrol can be observed providing complex mixtures of regioisomers (Scheme $1 \mathrm{~b}$ ).

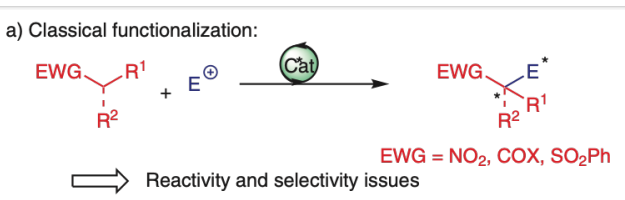

b) Classical ketone functionalization:

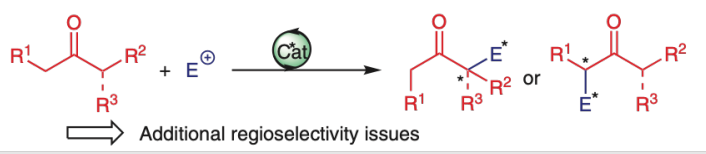

Scheme 1 Reactivity issues in classical functionalization

In order to find solutions to the challenge of eco-compatible enantioselective nucleophilic addition to an electrophile, chemists have invented a broad range of smart transient activation methods involving various pro-nucleophiles (Scheme 2).

For example, Mukaiyama-type activation of ketones through the generation of silyl enol ethers allows selective addition to a large range of electrophiles (Scheme 2a). ${ }^{2}$ However, this strategy requires initial insertion of the silyl group that is most of the time not recovered on the final product, thereby generating additional waste.

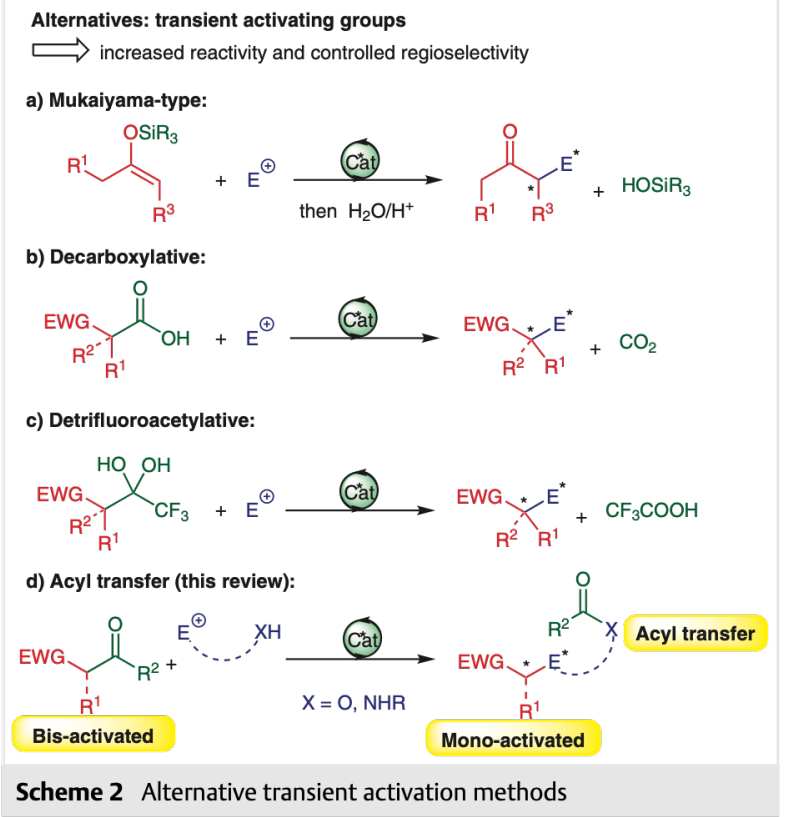

More recently, the application of decarboxylative additions has considerably broadened the range of possible enantioselective transformations (Scheme $2 b$ ). ${ }^{3}$ This approach is largely inspired by Nature where decarboxylative nucleophilic additions are at the center of polyketide synthesis. ${ }^{4}$ Taking advantage of such processes, chemists have devised innovative reactions starting from pro-nucleophiles activated through the insertion of a carboxylic acid, facilitating nucleophilic addition of the donor. ${ }^{5}$ The advantage of this approach lies in the mild reaction conditions generating only $\mathrm{CO}_{2}$ as the side product. However, the sometimes limited stability of the starting pro-nucleophiles might be problematic.

In recent years, a less general but promising detrifluoroacetylative approach has been reported (Scheme $2 \mathrm{c}$ ). ${ }^{6}$ Starting from a pro-nucleophile activated through the insertion of a fluorinated gem-diol, in situ detrifluoroacetylation generates a reactive enolate that is ready for trapping with a suitable electrophile. However, once again, the activating group is lost, this time in the form of $\mathrm{CF}_{3} \mathrm{COOH}$. In addition, the formation of this strong acid might be detrimental to many transformations or functional groups.

In order to combine the advantages of pro-nucleophile activation with enantioselective functionalization, an interesting solution is the acyl-transfer-based enantioselective strategy (Scheme 2d). ${ }^{6 a, 7}$ In this latter case, the insertion of a ketone on the pro-nucleophile considerably decreases the energy barrier for activation and facilitates selective addition to the electrophile. Further to this easier addition, the presence of an alcohol or amine function enables the transfer of the starting ketone (acyl transfer). This creates an additional valuable function on the final product while unveiling the starting desired mono-activated functional group. The latent nucleophilic handle required for the acyl transfer can be present at the onset (amine or alcohol functions) or can be generated during the first addition (for example, a transient enolate).

The general mechanism of the inter- or intramolecular acyl transfer strategy follows the reaction pathway shown in Scheme 3. First of all, catalytic activation triggers the addition of the pro-nucleophile to the appropriate electrophile. In most cases, formation of one or more stereogenic centers is controlled by the catalyst. The latent nucleophile $(\mathrm{XH})$ subsequently adds to the carbonyl function forming an acetal or hemiacetal triggering the spontaneous acyl transfer (Claisen-type fragmentation). The driving force for this fragmentation lies in the stabilization of the newly formed carbanion thanks to the presence of a strong electron-withdrawing substituent (EWG = ketone, nitro, sulfone, etc.). Protonation of this carbanion finally generates the desired products.

If the carbanion is trisubstituted ( $R^{1}$ is not $H$ ), a new stereogenic center is formed through this protonation, a challenging task in most processes. If another stereogenic center is present in the molecule, this process is diastereoselective and mostly under substrate control. However, the ease of deprotonation of the final adduct can lead to a thermodynamic equilibrium forming the most stable diastereomer. 


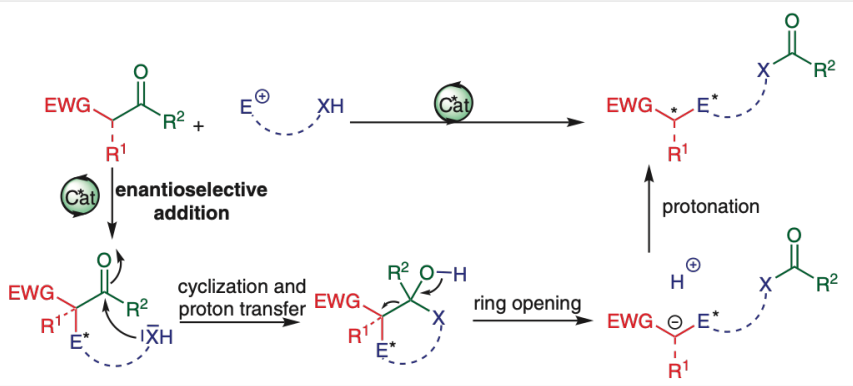

Scheme 3 General mechanism of addition followed by inter- or intramolecular acyl transfer

Alternatively, if the initial addition has not created any stereogenic center before the protonation (no-prochiral electrophile), the last step becomes the enantiodiscriminating one. As a result, a challenging appropriate catalyst-controlled enantioselective protonation is required for the formation of an enantioenriched final product.

As a result of this mechanism, the acyl transfer strategy is particularly well-suited for synthetic sequences involving challenging nucleophilic additions, as will be discussed during this overview. When the starting ketone is not embedded in a cycle, the strategy leads to the formation of linear products. In contrast, if the key starting ketone is embedded in a cycle, the acyl transfer strategy allows for a particular type of ring expansion, opening new opportunities for the formation of medium-sized rings.

\section{Intramolecular Acyl Transfer with Acyclic Substrates}

\subsection{Bifunctional Catalysis}

Given the ease of nucleophilic addition of pro-nucleophiles such as $\alpha$-nitroketones or $\beta$-dicarbonyl compounds upon organocatalytic activation, it is not surprising that some of the first examples of acyl-transfer-based enantioselective strategies employed this type of activation mode. For example, $\alpha$-nitroketones possess highly acidic protons resulting in easy deprotonation through the use of tertiary amines/Brønsted acid bifunctional catalysts.

In 2011, almost simultaneously, the groups of Yan, ${ }^{8}$ Wang, ${ }^{9}$ and Chan and Kwong ${ }^{10}$ reported the addition of $\alpha$ nitroketones 1 to $\beta, \gamma$-unsaturated $\alpha$-ketoesters 2 (Scheme 4). Yan showed that simple bifunctional tertiary aminethiourea catalyst cat1, derived from cyclohexane diamine, provided the expected acyl-protected enoates 3 with moderate to excellent enantiocontrol (62-97\% ee, Scheme 4a). ${ }^{8}$ The scope of the transformation is large with a limitation encountered using $\alpha$-substituted $\alpha$-nitroketones $\left(\mathrm{R}^{1}=\mathrm{al}\right.$ kyl), resulting in moderate diastereocontrol (around 2:1 dr). It is of great importance to point out that the two diastereomers are formed with different enantioselectivity. This in- dicates that this diastereoselectivity does not arise from thermodynamic control. Two different diastereomeric lactol intermediates formed with different enantiocontrol, leading to the formation of the two different diastereomers.
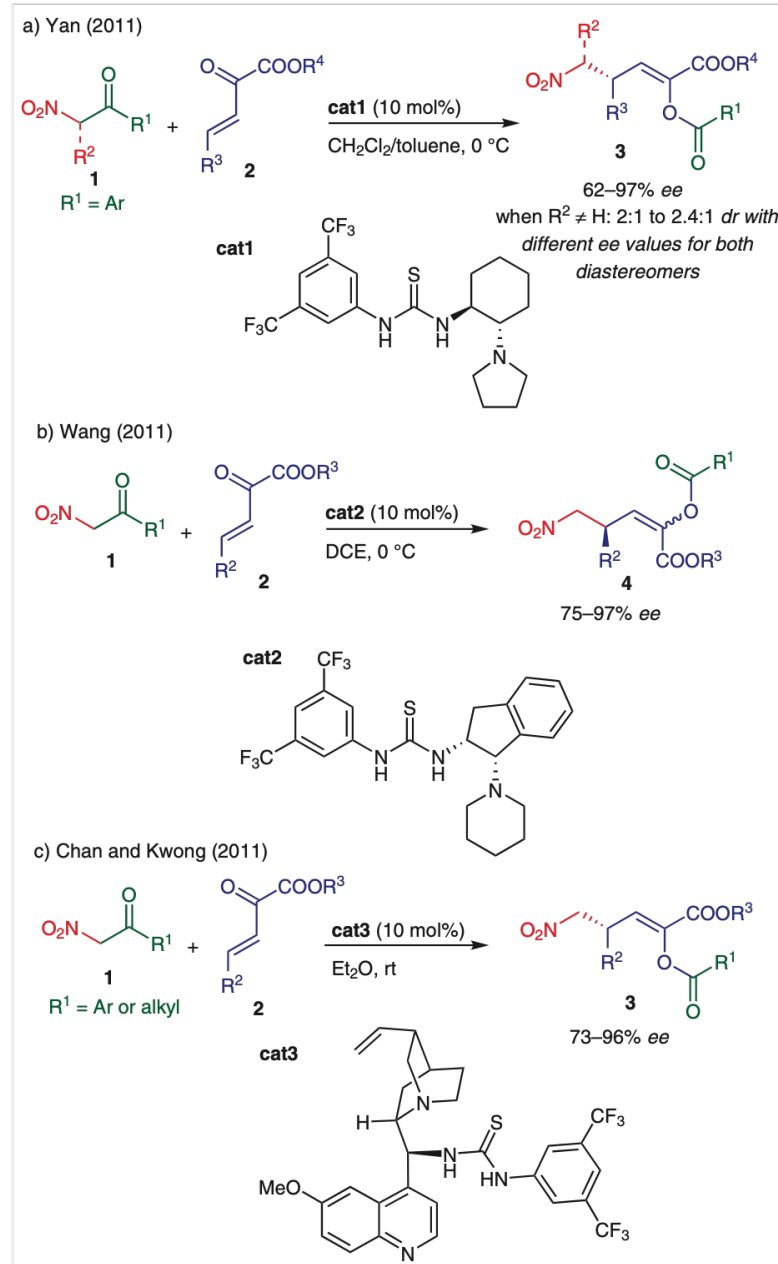

Scheme 4 Initial reports of an enantioselective intramolecular acyl transfer strategy 
Shortly after, the group of Wang showed that amino-indanol-derived bifunctional catalyst cat2 was also efficient in this transformation, providing the expected adducts 4 in $75-97 \%$ ee (Scheme $4 \mathrm{~b}$ ). ${ }^{9}$ The authors reported the formation of the E-enoates even though the mechanism of the transformation implies the formation of the Z-enoates (see the mechanism in Scheme 5).

Finally, in the same year, the group of Chan and Kwong reported the use of a quinine-derived bifunctional thiourea catalyst cat 3 for the same reaction leading to enoates $\mathbf{3}$ in $73-96 \%$ ee (Scheme $4 \mathrm{c}$ ). ${ }^{10}$ The organocatalyst enabled the successful use of aliphatic $\alpha$-nitroketones $\mathbf{1}\left(\mathbf{R}^{1}=\right.$ alkyl).

Of particular interest, in all these transformations, the direct use of nitromethane or other nitroalkanes only provided trace amounts of the corresponding Michael adducts. This highlights the interest of the strategy where the use of the $\alpha$-nitroketones 1 favors the Michael addition under mild conditions and with excellent enantiocontrol. The mechanism of this transformation implies Michael addition forming an enolate that is directly trapped through hemiacetal formation (Scheme 5). Subsequent ring opening provides the protected enoate 3 with $Z$-configuration.

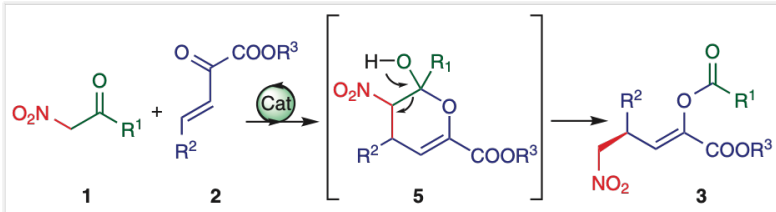

Scheme 5 Mechanism of the addition of $\alpha$-nitroketones to $\beta, \gamma$-unsaturated $\alpha$-ketoesters

Inspired by this pioneering use of $\alpha$-nitroketones, in 2013 , the group of Wang and Zhou found that $\beta$-dicarbonyl compounds 6 and functionalized nitroolefins 7 could be condensed in the presence of a bifunctional tertiary aminesquaramide catalyst cat4. The presence of a phenol on the nitroolefin, triggered the acyl transfer providing 5-nitro-2oxo-4-arylpentanoates 8 in $80-98 \%$ ee (Scheme 6). ${ }^{11}$ Once again, the use of activated dicarbonyl pro-nucleophiles facilitates the initial enantioselective Michael addition leading to a highly defined transition state through multiple activation of both the $\beta$-dicarbonyl compound and nitroolefin, which explains the excellent enantiocontrol observed.

Following this report, the group of $\mathrm{Xu}$ and Wang disclosed the challenging preparation of oxindoles $\mathbf{1 0}$ through an acyl transfer strategy (Scheme 7). ${ }^{12}$ This approach condensing 3-acetyl-oxindoles 9 with $\beta, \gamma$-unsaturated ketoesters $\mathbf{2}$ involves a diastereoselective protonation forming the oxindole stereocenter. Even though the quinine-derived bifunctional catalyst cat5 is able to efficiently control the newly formed stereocenter on the Michael adduct (up to $90 \% e e$ ), the diastereoselective protonation results in moderate control (up to 77:23 dr). Once again, the observation

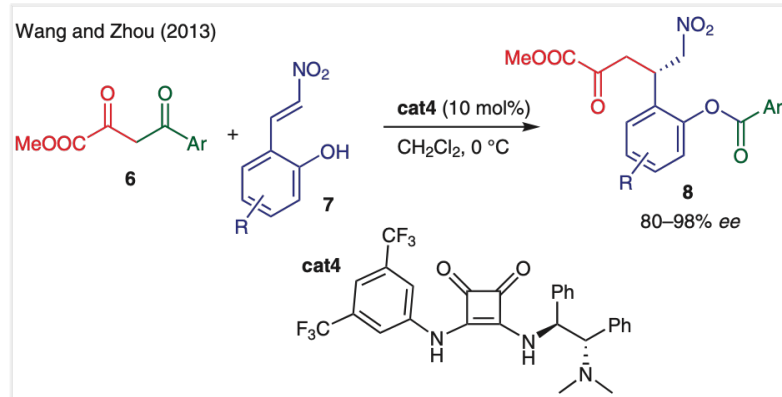

Scheme 6 Formation of 5-nitro-2-oxo-4-arylpentanoates via an acyl transfer strategy

of different enantioselectivities between the different diastereomers indicates a kinetic control from two different diastereomeric intermediates.

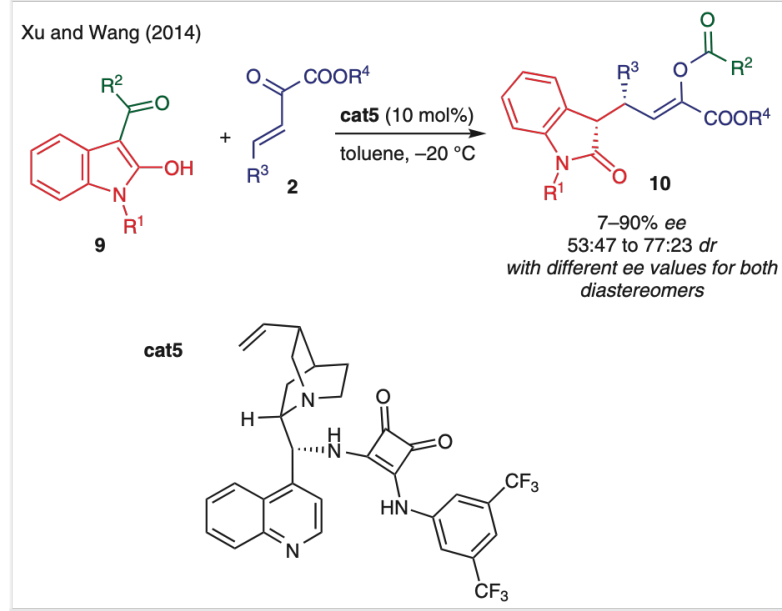

Scheme 7 Formation of chiral oxindoles by an acyl transfer strategy

In 2016, our group took advantage of the addition of $\beta$ diketones 11 to hydroxy-nitroolefins 12 to prepare enantioenriched ketones 13, illustrating the power of the acyl transfer strategy (Scheme 8). ${ }^{13}$ The direct addition of mono-ketones to substituted nitroolefins results in particularly low reactivity ( 36 to 288 hours), and limited scope and enantiocontrol. The solution to this reactivity challenge lies in the use of $\beta$-dicarbonyl pro-nucleophiles such as the $\beta$ diketones 11 reported by the group of Enders. ${ }^{14}$ The resulting Michael adducts are readily formed with excellent enantiocontrol using a bifunctional quinine catalyst (up to $96 \%$ ee). However, the hemiacetal intermediates were found to be reluctant to fragmentation, instead leading to dehydration. The solution to this drawback was the discovery that the use of simple catalytic $\mathrm{Fe}(\mathrm{CO})_{5}$ upon light activation promoted the selective desired acyl transfer. The in situ generated iron complex must act as a mild Lewis acid po- 
tentially activating either the dicarbonyl, the nitro group or both functions to facilitate the fragmentation. The obtained compounds 13 are valuable multi-functionalized building blocks of interest. To highlight this potential, reduction of the nitro group in $\mathbf{1 4}$ was shown to directly afford valuable substituted pyrrolidines 15 (Scheme 8).
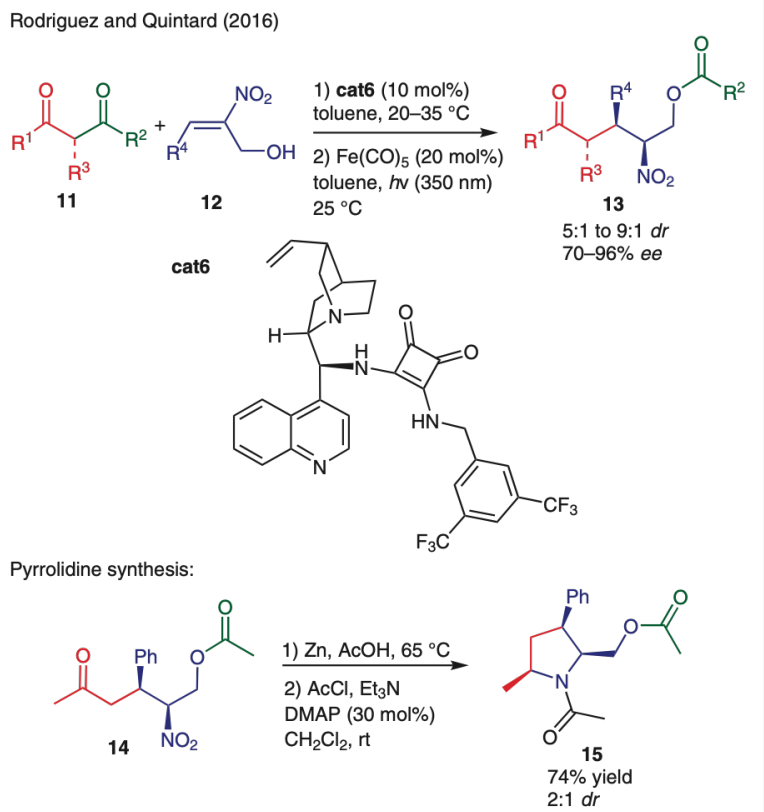

Scheme 8 Multi-catalyzed Michael addition-acyl transfer

More recently, Pan's group increased the scope of these organocatalytic acyl transfer transformations with different strategies. Taking advantage of the already reported excellent reactivity of $\alpha$-nitroketones $\mathbf{1}$, a bifunctional organocatalyst could promote the addition of this class of compound to unsaturated pyrazolones 16 (Scheme 9). ${ }^{15}$ The modified bifunctional tertiary amine-thiourea organocatalyst cat7 controlled the enantioselectivity of the newly formed stereocenter with excellent efficiency (up to $99 \%$ $e e)$. The generation of the enolate triggers the fragmentation, unveiling the nitroalkane and protecting the pyrazolone as the 3-acyloxy-pyrazole 17. Post-transformation of the obtained adduct indicated that the nitro group can be easily reduced to the corresponding amine.

Continuing their interest on this approach, the same group recently took advantage of the activation of $\beta$-dicarbonyl compounds 11 by a quinine-derived bifunctional catalyst cat8, inducing the addition to nitroolefins 7 possessing phenol substituents (Scheme 10). ${ }^{16}$ This process is an extension of the chemistry developed by Wang and Zhou (see Scheme 6) ${ }^{11}$ using the parent 1,3-diketones. Formation of the Michael adduct initiates the fragmentation transferring one of the two ketones to protect the phenol while unveiling a mono-ketone on the other side. As a result of the

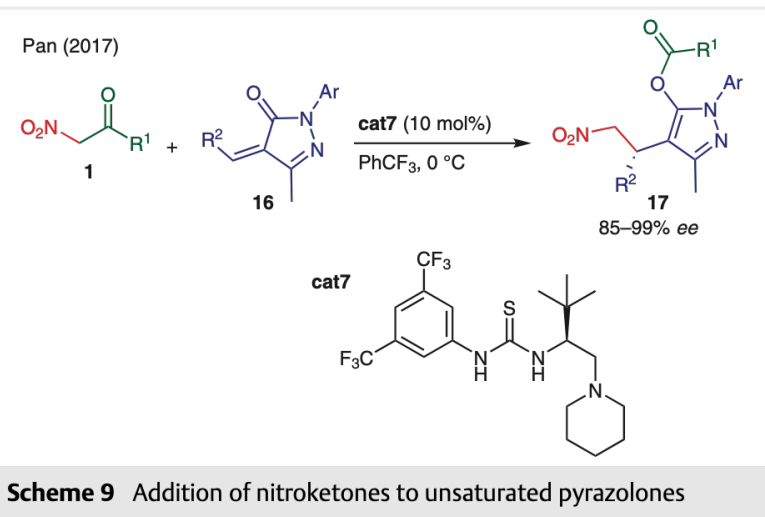

bis-activation of the pro-nucleophile, the reaction occurs selectively under mild conditions providing the final products in 74 to $97 \%$ ee. Of interest, starting from a disymmetric diketone, selective migration of the acetyl group is preferred over migration of the aromatic ketone.

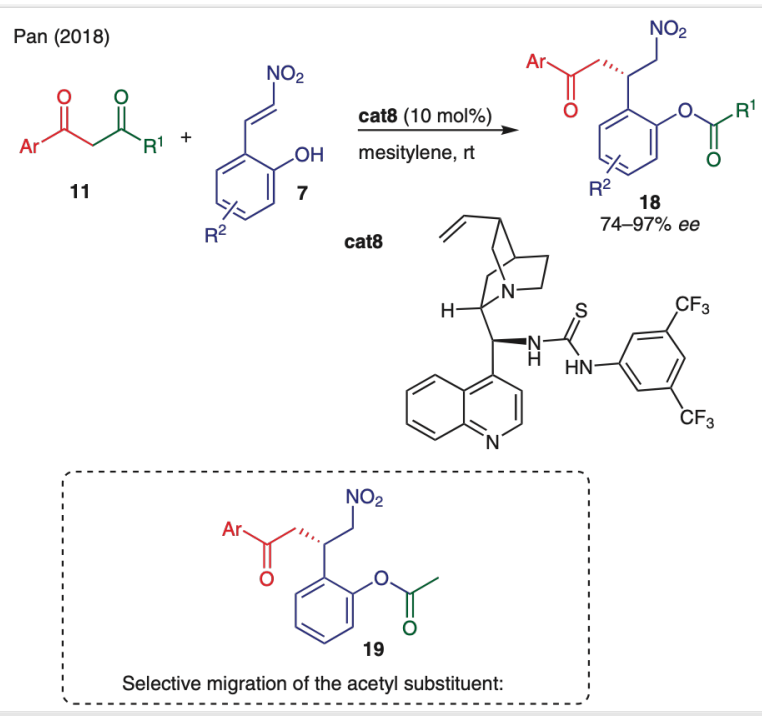

Scheme 10 Addition of diketones to hydroxy-containing nitroolefins

Further extending the scope of acyl transfer strategies, Pan's group recently reported the addition of nitroketones 1 to enones $\mathbf{2 0}$ and $\mathbf{2 1}$ possessing a terminal alcohol to trigger the final acyl transfer (Scheme 11). ${ }^{17}$ Starting from the $\alpha$ nitroketone was crucial to achieve this process since control experiments indicated that nitromethane was not reactive enough to promote the Michael addition. Once again, the ketone activates the Michael donor while directly protecting the final hydroxy function. The use of the quinine-derived squaramide organocatalyst cat9 provided optimized enantiocontrol in this transformation. Derivatization of the products could be performed by nitro group reduction providing interesting substituted pyrrolidines 25 . 


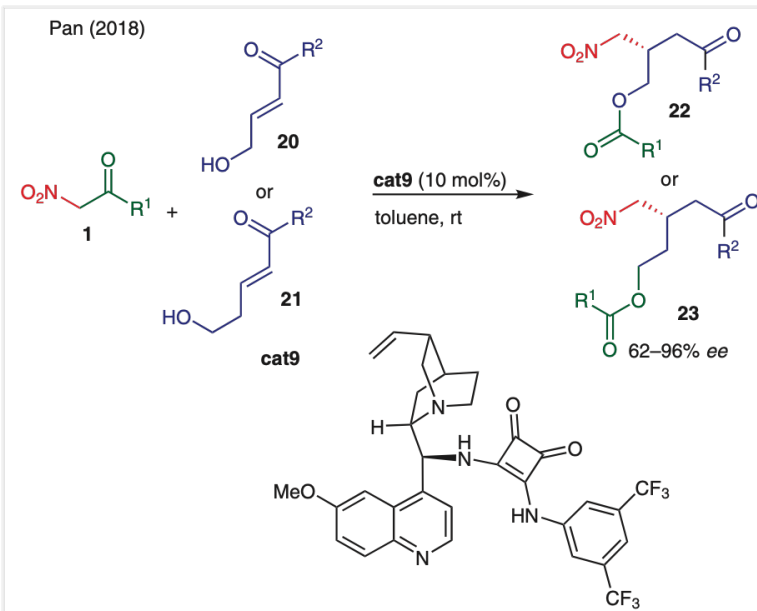

Application in pyrrolidine synthesis:

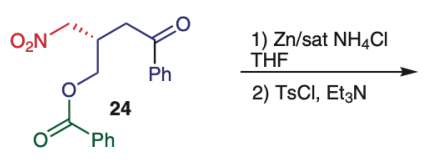

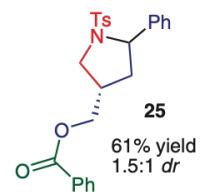

Scheme 11 Addition of nitroketones to enones

With an appropriate tool to activate $\alpha$-nitroketones, the scope was extended to the addition to in situ generated ortho-quinone methides (Scheme 12). ${ }^{18}$ Generation of this reactive species from 26 by $\mathrm{NaHCO}_{3}$-promoted sulfone elimination induces the subsequent catalyzed Michael addition, affording a phenol able to promote the acyl transfer. As observed for other transformations, control experiments with nitroalkane pro-nucleophiles did not give any Michael addition, which serves to highlight the interest of the approach. The appropriate use of the bifunctional catalyst provides excellent enantioselectivities in this reaction (up to $98 \% e e)$.

\subsection{Aminocatalysis}

Secondary or primary amine organocatalysts are particularly useful thanks to their ability to selectively activate carbonyl compounds. ${ }^{19}$ However, one of the challenges encountered in this field is the generation and transformation of these carbonyl functions. In order to solve this problem, our group reported that efficient merging between ironand organocatalytic cycles allowed the addition of $\beta$-diketones $\mathbf{1 1}$ to allylic alcohols $\mathbf{2 8}$ triggering a final acyl transfer (Scheme 13).20

Reversible hydrogen transfer (borrowing hydrogen) from the alcohol 28 to the iron complex induces the formation of a catalytic amount of $\alpha, \beta$-unsaturated aldehyde 30 required for the aminocatalytic cycle. ${ }^{21}$ Reaction of this al-

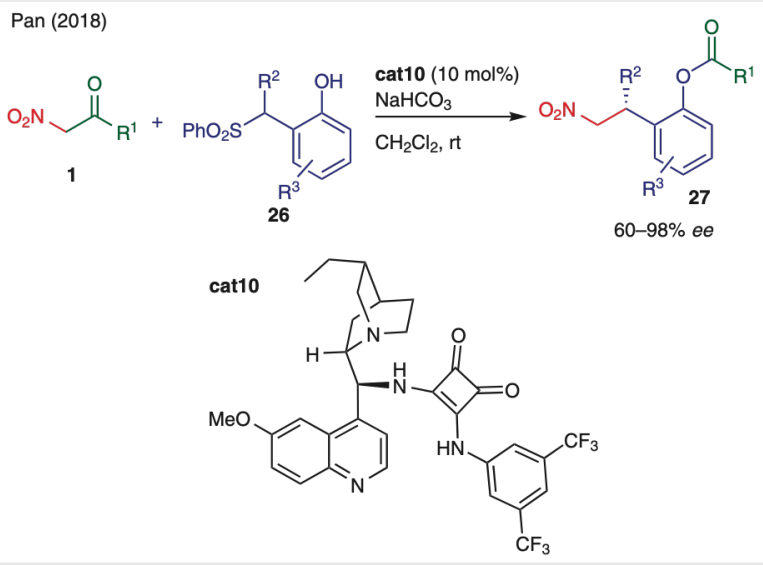

Scheme 12 Addition to in situ generated ortho-quinone methides

dehyde through dual activation by the aminocatalyst cat11 and a copper co-catalyst (activating the $\beta$-diketone) controls the enantioselectivity of the process (up to $96 \% \mathrm{ee}$ ). ${ }^{22}$ As a result, this multi-catalytic approach allows the direct enantioselective addition of a Michael donor to allylic alcohols. Once again, the second ketone on the pro-nucleophile facilitates the Michael addition while directly protecting the hydroxy function through the cascade.

A broad range of different aliphatic and aromatic $\beta$ diketones can be used efficiently in this transformation highlighting its great tolerance. Most notably, starting from dissymmetrical $\beta$-diketones such as $\mathbf{3 3}$, selective migration of the acetyl group was observed in the presence of a cyclic ketone (Scheme 14a).

However, in a competition between two acyclic ketones as in 36, a 2:1 selectivity of the migration of the acetyl group is observed. Of importance, the two products 37a and 37b are obtained with different enantiocontrol indicating that they potentially arise from different reaction intermediates such as two diastereomers (Scheme 14b).

The potential selective migration of an acetyl function could be advantageously applied in the efficient construction of different natural products or fragments of interest, for example, in the formal synthesis of apratoxin A (Scheme 15). ${ }^{20}$ This synthetic application is interesting because it demonstrates the value of this acyl transfer strategy in activating poor nucleophiles. Triggered by the iron-catalyzed borrowing-hydrogen, organocatalytic addition of the $\beta$ diketone $\mathbf{3 8}$ to crotyl alcohol (34) is followed by the acyl transfer unveiling the desired challenging tert-butyl ketone 39. Further reduction furnishes the enantiopure diol $\mathbf{4 0}$ in only two steps from commercial products, a considerably shorter route than the previously described 6- to 12-step sequences. 


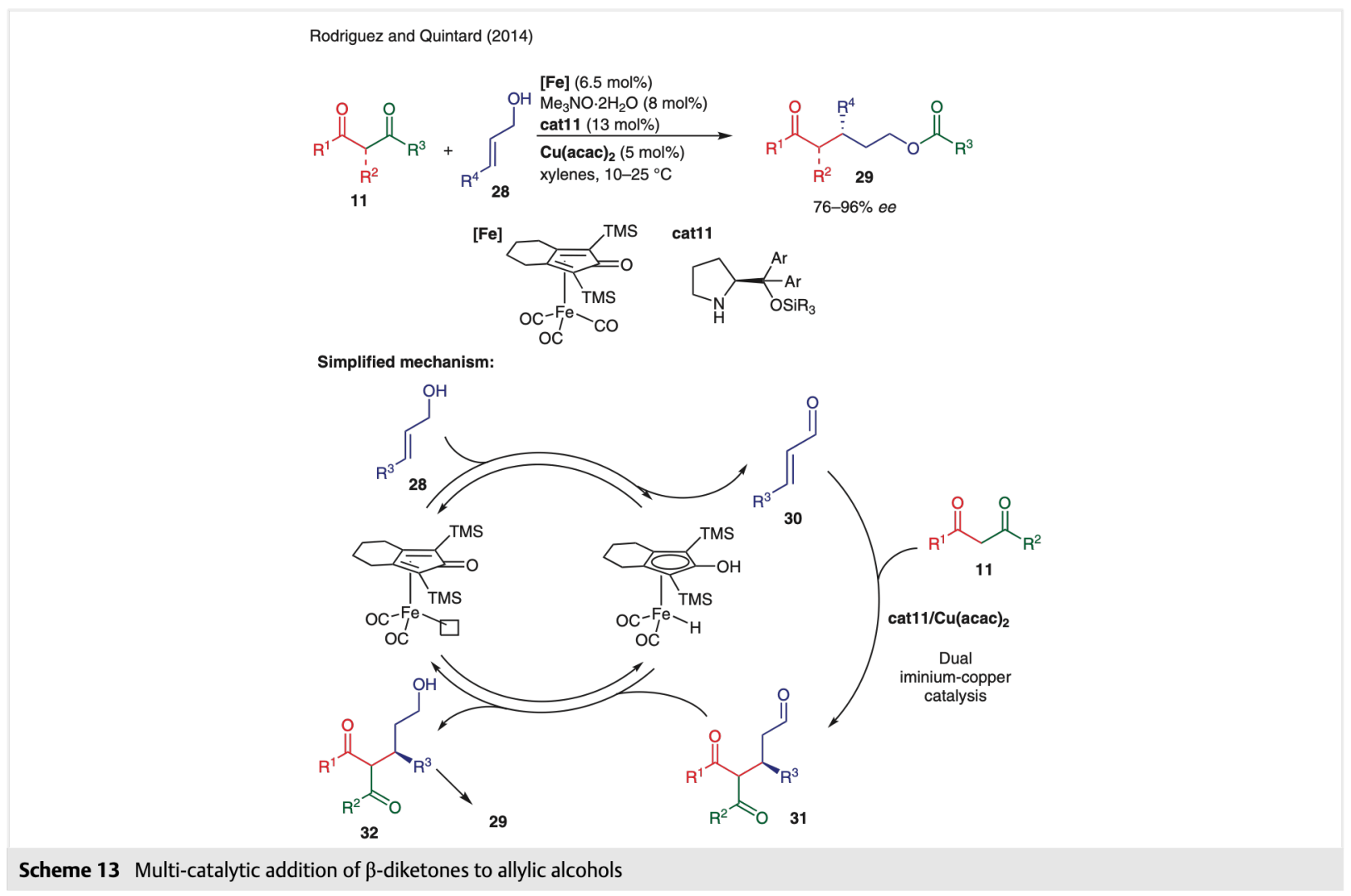

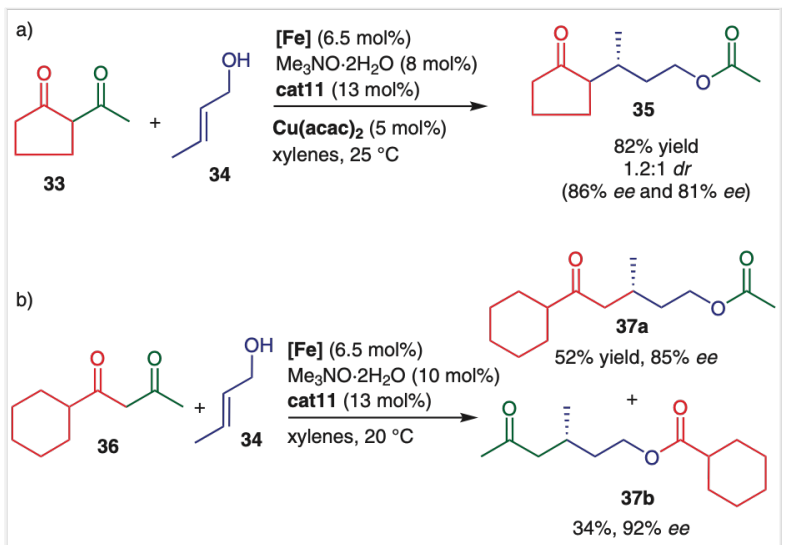

Scheme 14 Multi-catalytic addition of $\beta$-diketones to crotyl alcohol

More recently, the scope of the multi-catalytic cascade was extended to the use of $\beta$-ketosulfones 41 (Scheme 16). ${ }^{22,23}$ Using the same iron-catalyzed borrowing hydrogen approach, addition to crotyl alcohol through a dual copperiminium activation furnished the desired alcohols with

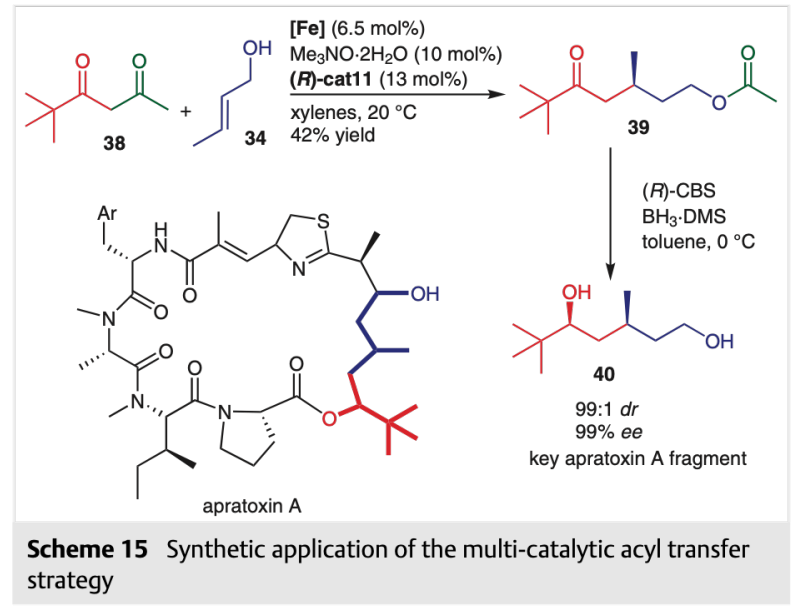

moderate enantiocontrol ( $68 \%$ ee). Using this substrate, the acyl transfer did not occur spontaneously but required heating at $100{ }^{\circ} \mathrm{C}$ to produce the linear sulfone and protected alcohol function. 
Rodriguez and Quintard (2016)

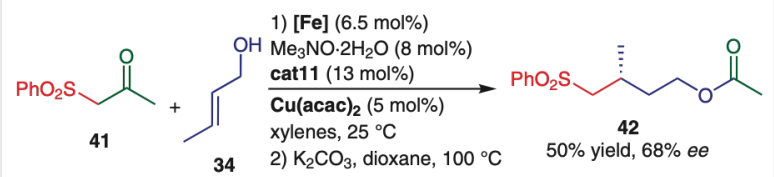

Scheme 16 Use of a ketosulfone in the multi-catalytic addition to crotyl alcohol

In 2016, the group of Luo reported an interesting strategic use of acyl transfer in the addition of $\beta$-diketones $\mathbf{1 1}$ to in situ generated ortho-quinone methide $45 .{ }^{24}$ In this transformation, the use of the primary amine organocatalyst cat12, which was able to selectively activate the $\beta$-diketone, was crucial in generating the final product $\mathbf{4 4}$ in typically high enantiocontrol through a rare example of enantioselective protonation (up to $98 \%$ ee) (Scheme 17).

$$
\text { Luo (2016) }
$$

Scheme 17 Amino-catalyzed addition of $\beta$-diketones to ortho-quinone methide

At the onset, from 43, the use of KF liberates the reactive ortho-quinone methide $\mathbf{4 5}$ (Scheme 18). After amino-catalyzed Michael addition, hemiacetal formation followed by Claisen fragmentation liberates an enamine 48. This key intermediate leads to the formation of the chiral ketone $\mathbf{4 4}$ through enantioselective protonation. For this purpose, the role of the acid co-catalyst is crucial to obtain a well-defined transition state and an optimum level of enantiocontrol. It must be pointed out that the final ketone is stable under the reaction conditions without racemization through enamine back formation or deprotonation.

More recently, the group of Pan demonstrated that upon iminium activation by a secondary amine organocatalyst cat11, $\alpha, \beta$-unsaturated aldehydes $\mathbf{4 9}$ underwent addition of $\alpha$-nitroketones 1 to afford, after acyl transfer, 2,4-disubstituted chromane derivatives $\mathbf{5 0}$ (Scheme 19). ${ }^{25}$ The secondary amine catalyst is able to selectively construct the benzylic stereocenter while the irreversible fragmentation on the strained ketal accounts for the excellent diastereoselectivity of the reaction ( $>20: 1 d r$ and up to $96 \% e e$ ). The acyl transfer constitutes a driving force for the reaction since it was previously shown that under the same conditions addition of $\alpha$-nitroketones to $\alpha, \beta$-unsaturated aldehydes with-
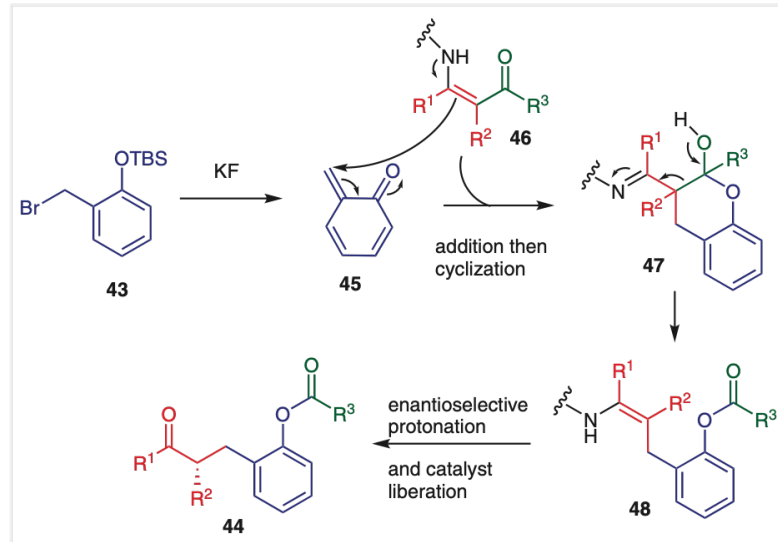

Scheme 18 Mechanism of enantiocontrol in the addition of $\beta$-diketones to ortho-quinone methides

out the phenol substituent did not induce the corresponding Michael addition. ${ }^{8}$ Reduction of the obtained adduct provided an interesting entry into 4 -substituted chromane derivatives.

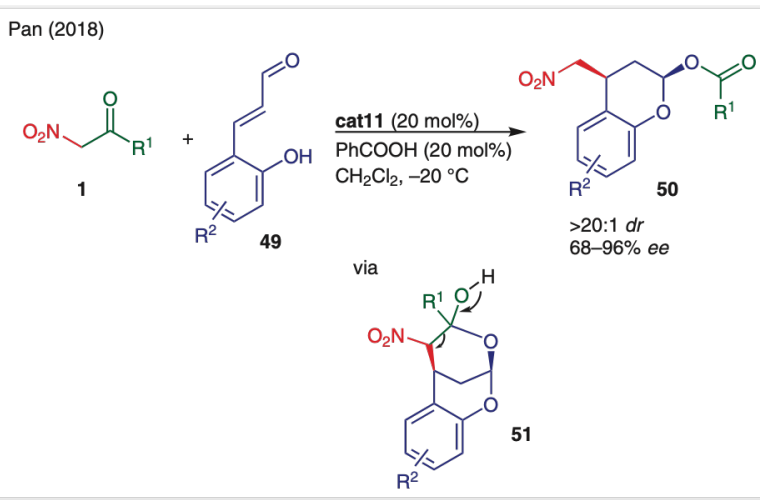

Scheme 19 Addition of $\alpha$-nitroketones to $\alpha, \beta$-unsaturated aldehydes

\section{Intermolecular Acyl Transfer with Acyclic Substrates}

The acyl transfer strategy has also been applied to initiate enolate generation in the acyclic series. In this case, the initial ketone is lost during the process. In 2016, the team of Yi and Zhang reported the use of the bifunctional organocatalyst cat 3 for the in situ generation of reactive bis-fluorinated enolates 56 (Scheme 20). ${ }^{26}$ Starting from a bis-fluorinated $\beta$-diketone $\mathbf{5 2}$, in the presence of the organocatalyst and methanol, initial acyl transfer generates the otherwise difficult to prepare bis-fluorinated enolates 56 . These nucleophiles subsequently add to protected isatins $\mathbf{5 3}$ creating challenging tetrasubstituted carbon centers with excellent enantiocontrol (up to $98 \%$ ee). Control experiments indicated that monofluorinated $\beta$-diketones are inefficient in this 


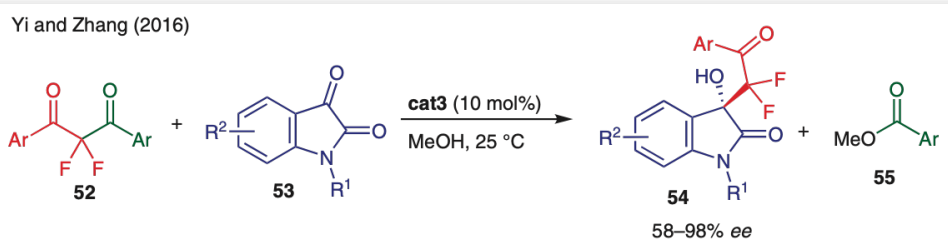

In situ enolate generation:

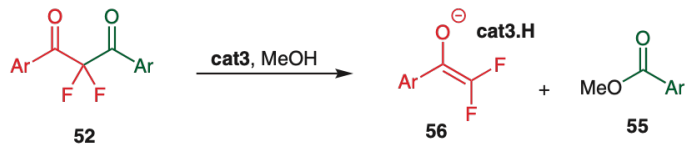

Scheme 20 Organocatalytic intermolecular acyl transfer strategy

process as well as a direct aldolization from the corresponding mono-ketone, highlighting the interest of the intermolecular acyl transfer approach.

Despite the considerable amount of known transformations utilizing metal catalysts, enantioselective acyl transfer strategies have been scarcely applied using this type of catalytic activation mode. The group of Tunge has demonstrated the great potential of using palladium catalysis in deacylative transformations, notably in the achiral or racemic series. $^{27}$ In 2013, the same group developed the first enantioselective variant of this approach involving the condensation of $\beta$-diketones $\mathbf{5 7}$ with allylic alcohols $\mathbf{5 8}$ (Scheme 21). ${ }^{28}$

The process is initiated by the addition of the sodium alcoholate to the $\beta$-diketone $\mathbf{5 7}$ liberating a reactive enolate 61 and an allylic acetate $\mathbf{6 2}$ (Scheme 21). This allylic acetate is able to undergo activation by the palladium complex generating the required $\pi$-allyl complex necessary for the eno- late trapping. The use of a PHOX-type ligand controls the stereoselectivity of the addition forming challenging quaternary stereocenters in up to $95 \%$ ee.

This transformation was further applied in the formal synthesis of (+)-hamigeran B based on fragment 65 previously disclosed by Stoltz (Scheme 22). ${ }^{29}$

Finally, a two-step acyl transfer has also been used to favor an enantioselective allene functionalization. In 2017, the group of Breit took advantage of the reactivity of $\beta$ diketones 11 to propose a two-step addition to allenes 66 followed by deacylation (Scheme 23). ${ }^{30}$ The interest behind the use of $\beta$-diketones lies in their better reactivity and the selectivity of their addition as compared to simple ketones. As a result, the overall addition-deacylation products 67 can be obtained easily and with good enantiocontrol through the use of a rhodium complex in combination with a chiral phosphoramidite ligand (up to $95 \% e e$ ).

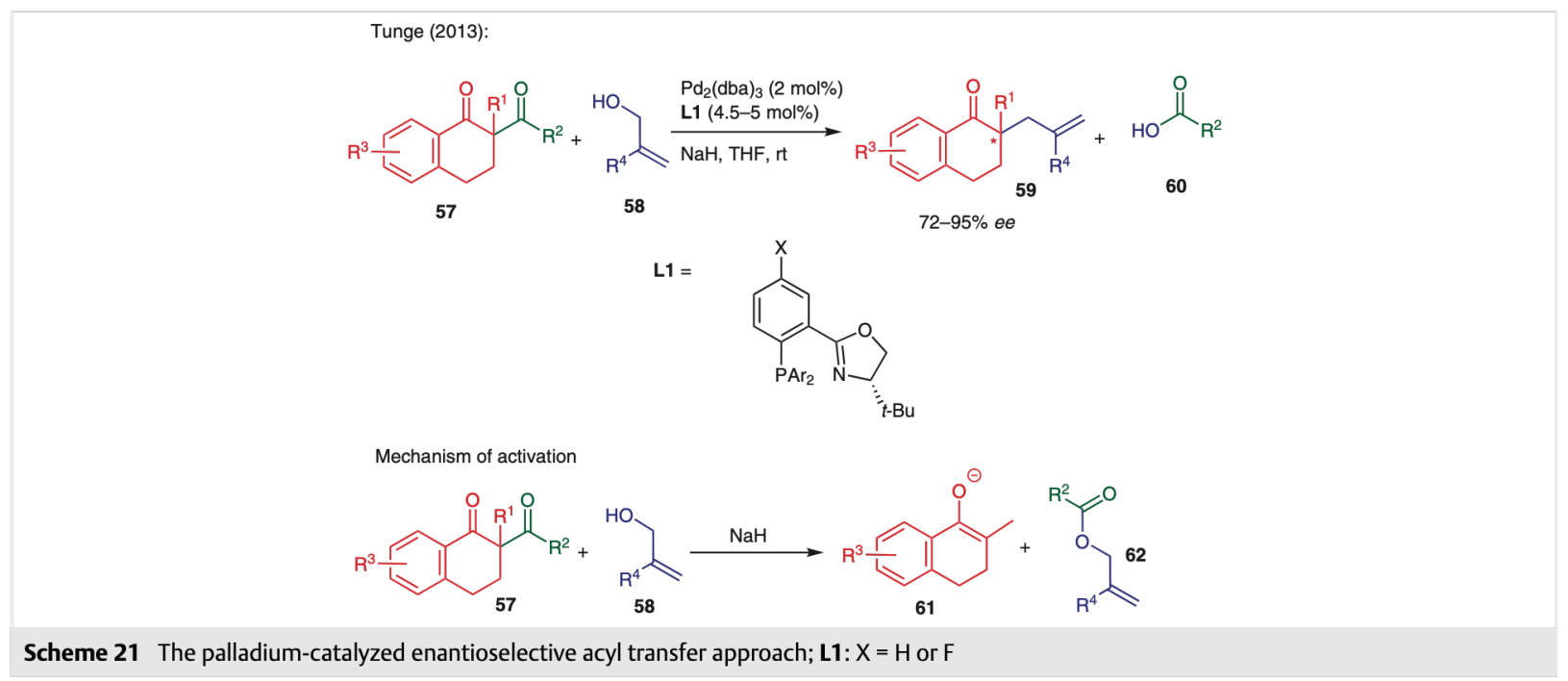




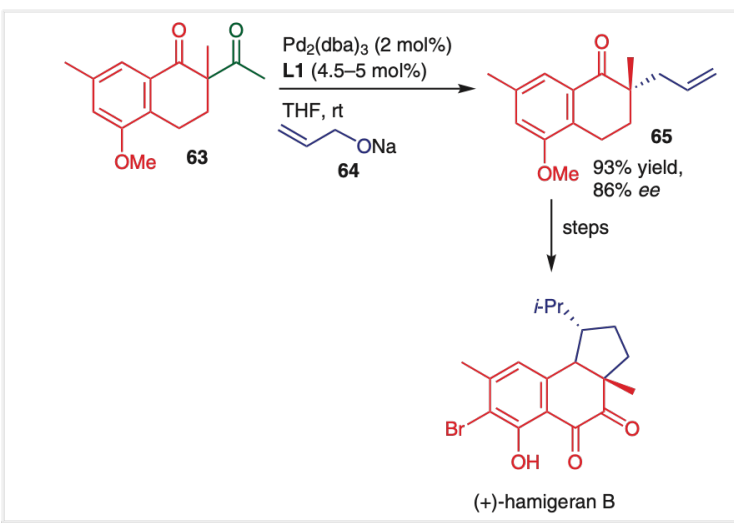

Scheme 22 Formal synthesis of (+)-hamigeran B

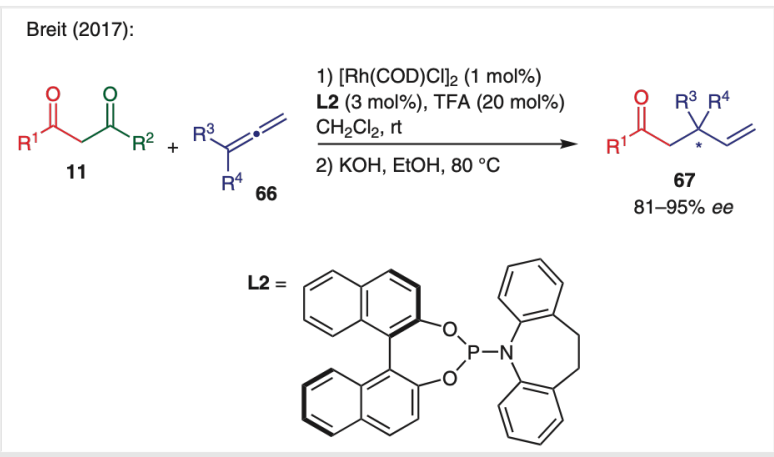

Scheme 23 Rhodium-catalyzed allene functionalization

\section{Medium-Sized-Ring Formation with Cyclic Substrates}

Claisen-type fragmentations have been commonly used for the preparation of valuable but challenging to prepare medium-sized-ring lactones. ${ }^{31}$ In order to assemble easily and enantioselectively the precursor hemiacetals required for the fragmentation, our group developed a strategy based on an initial Michael addition of $\beta$-diketones 68 to $\alpha, \beta$-unsaturated aldehydes $\mathbf{3 0}$ upon iminium activation with cat11 (Scheme 24 ). ${ }^{32}$ From the initial enantioenriched hemiacetals, reduction followed by heating at $100^{\circ} \mathrm{C}$ provided the desired lactones 70 in $91-99 \%$ ee. It was found that the role of the base in the fragmentation was not to assist deprotonation of the alcohol but to remove any potential trace of acid leading to an undesired dehydration. Through this strategy different 10 -membered ring lactones can be prepared in only three steps. In the case of an 11membered ring lactone, the fragmentation occurs spontaneously at room temperature highlighting the role of the hemiacetal geometry over ring expansion kinetics.

Taking advantage of this strategy, the group of Rodriguez and Coquerel recently extended the ring expansion to the formation of 8-membered ring lactams 73 (Scheme 25). ${ }^{33}$ This reaction is different from the previous examples since nitrogen is involved in the intramolecular ring fragmentation providing the desired lactam. Starting from a cyclobutanone $\beta$-ketoamide 71, Michael addition on 72 using bifunctional quinine-derived squaramide cat 6 followed by the ring expansion formed the lactams with excellent enantiocontrol (up to $99 \%$ ee). The diastereoselectivity of the transformations are moderate $(5: 1$ to $15: 1 d r)$ and might originate from thermodynamic control.

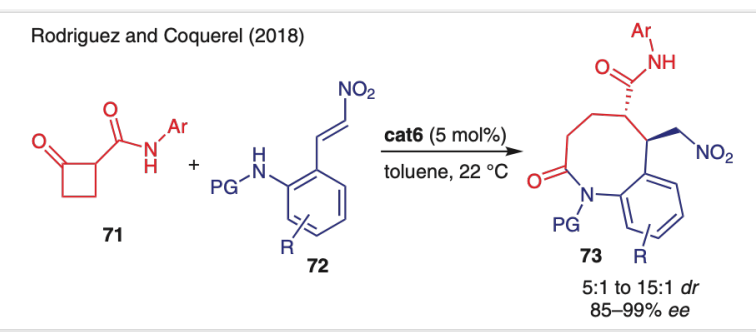

Scheme 25 Medium-sized-ring lactam formation employing an acyl transfer strategy

\section{Conclusion}

In conclusion, over the years, enantioselective acyl transfer strategies have found broad applications in organic synthesis. The methods developed take advantage of the higher reactivity and selectivity of activated pro-nucleophiles such as $\alpha$-nitroketones, $\beta$-dicarbonyl compounds and $\beta$-ketosulfones to promote a broad range of catalytic transformations. The presence of a latent hydroxy or nitrogen function induces a fragmentation unveiling one of the two functions from the starting pro-nucleophile, while the oth-

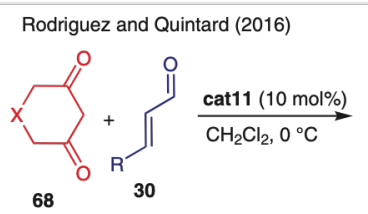<smiles></smiles>

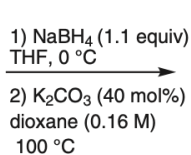

$100^{\circ} \mathrm{C}$

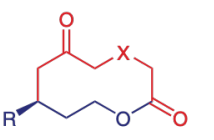

70

$91-99 \%$ ee

10 - or 11 -

lactones
lambered

Scheme 24 Medium-sized-ring formation employing an acyl transfer strategy 
er is transferred to directly serve as a new functional group (ester, amide) or as a protecting group. The new reactivity opportunities as well as the high levels of stereocontrol traditionally observed should inspire organic chemists to find innovative solutions in the smart activation of a broad range of organic molecules. Given the power of metal catalysis, a growing number of acyl transfer transformations involving metal complexes should be discovered. In addition, the future should see an increase in the synthetic applications of these strategies thanks to the considerable synthetic economies these methods generate.

\section{Funding Information}

The Centre National de la Recherche Scientifique (CNRS) and AixMarseille Université are gratefully acknowledged for financial support.

\section{References}

(1) (a) Hendrickson, J. B. J. Am. Chem. Soc. 1975, 97, 5784. (b) Trost, B. M. Science 1991, 254, 1471. (c) Wender, P. A.; Miller, B. L. Nature 2009, 460, 197. (d) Gaich, T.; Baran, P. S. J. Org. Chem. 2010, 75, 4657.

(2) For reviews, see: (a) Beutner, G. L.; Denmark, S. E. Angew. Chem. Int. Ed. 2013, 52, 9086. (b) Kan, S. B. J.; Ng, K. K.-H.; Paterson, I. Angew. Chem. Int. Ed. 2013, 52, 9097. (c) Sutar, R. L.; Joshi, N. N. Tetrahedron: Asymmetry 2013, 24, 1345. (d) Kitanosono, T.; Kobayashi, S. Adv. Synth. Catal. 2013, 355, 3095. (e) For a pioneering contribution, see: Mukaiyama, T.; Banno, K.; Narasaka, K. J. Am. Chem. Soc. 1974, 96, 7503.

(3) For reviews, see: (a) Pan, Y.; Tan, C.-H. Synthesis 2011, 2044. (b) Wang, Z.-L. Adv. Synth. Catal. 2013, 355, 2745. (c) Nakamura, S. Org. Biomol. Chem. 2014, 12, 394. For pioneering contributions, see: (d) Lalic, G.; Aloise, A. D.; Shair, M. D.J. Am. Chem. Soc. 2003, 125, 2852. (e) Magdziak, D.; Lalic, G.; Lee, H. M.; Fortner, K. C.; Aloise, A. D.; Shair, M. D. J. Am. Chem. Soc. 2005, 127, 7284. (f) Lubkoll, J.; Wennemers, H. Angew. Chem. Int. Ed. 2007, 46, 6841. (g) Ricci, A.; Pettersen, D.; Bernardi, L.; Fini, F.; Fochi, M.; Perez Herrera, R.; Sgarzani, V. Adv. Synth. Catal. 2007, 349, 1037. (h) Yin, L.; Kanai, M.; Shibasaki, M. J. Am. Chem. Soc. 2009, 131, 9610. (i) Bae, H. Y.; Sim, J. H.; Lee, J. W.; List, B.; Song, C. E. Angew. Chem. Int. Ed. 2013, 52, 12143. (j) Quintard, A.; Rodriguez, J. Chem. Eur. J. 2015, 21, 14717. (k) Saadi, J.; Wennemers, H. Nat. Chem. 2016, 8, 276. (1) Quintard, A.; Rodriguez, J. ACS Catal. 2017, 7, 5513. (m) Akamatsu, M.; Sakai, N.; Matile, S. J. Am. Chem. Soc. 2017, 139, 6558.

(4) For reviews, see (a) Hill, A. Nat. Prod. Rep. 2006, 23, 256. (b) Staunton, J.; Weissman, K. J. Nat. Prod. Rep. 2001, 18, 380.

(5) Alternatively, decarboxylative transformations can be performed from activated esters (palladium-catalyzed allylation for example), see: (a) Tunge, J. A.; Burger, E. O. Eur. J. Org. Chem. 2005, 1715. (b) Weaver, J. D.; Recio, A. III.; Grenning, A. J.; Tunge, J. A. Chem. Rev. 2011, 111, 1846.

(6) For pioneering results, see: (a) Han, C.; Kim, E. H.; Colby, D. A. J. Am. Chem. Soc. 2011, 133, 5802. (b) Saidalimu, I.; Fang, X.; He, X.-P.; Liang, J.; Yang, X.; Wu, F. Angew. Chem. Int. Ed. 2013, 52, 5566. (c) Zhang, P.; Wolf, C. Angew. Chem. Int. Ed. 2013, 52 , 7869. (d) Xie, C.; Wu, L.; Han, J.; Soloshonok, V. A.; Pan, Y. Angew. Chem. Int. Ed. 2015, 54, 6019.
(7) For reviews, see: (a) Castro, A. M. M. Chem. Rev. 2004, 104, 2939. (b) Ortega-Martínez, A.; Molina, C.; Moreno-Cabrerizo, C.; Sansano, J. M.; Nájera, C. Eur. J. Org. Chem. 2018, 2394. For nonenantioselective examples, see: (c) Ballini, R.; Petrini, M.; Polimanti, O. J. Org. Chem. 1996, 61, 5652. (d) Giorgi, G.; Arroyo, F. J.; Lopez-Alvarado, P.; Menendez, J. C. Synlett 2010, 2465. (e) Giorgi, G.; Arroyo, F. J.; Lopez-Alvarado, P.; Menendez, J. C. Tetrahedron 2011, 67, 5582. (f) Richter, C.; Voigt, B.; Mahrwald, R. RSC Adv. 2015, 5, 45571. (g) Ding, R.; Wolf, C. Chem. Commun. 2016, 52, 3576. (h) Ortega-Martínez, A.; Molina, C.; MorenoCabrerizo, C.; Sansano, J. M.; Nájera, C. Synthesis 2017, 49, 5203.

(8) Lu, R.-J.; Yan, Y.-Y.; Wang, J.-J.; Du, Q.-S.; Nie, S.-Z.; Yan, M.J. Org. Chem. 2011, 76, 6230 .

(9) Gao, Y.; Ren, Q.; Siau, W.-Y.; Wang, J. Chem. Commun. 2011, 47, 5819.

(10) Li, P.; Chan, S. H.; Chan, A. S. C.; Kwong, F. Y. Org. Biomol. Chem. 2011, 9, 7997.

(11) Liu, Y.; Wang, Y.; Song, H.; Zhou, Z.; Tang, C. Adv. Synth. Catal. 2013, 355, 2544.

(12) Zhou, J.; Jia, L.-N.; Wang, Q.-L.; Peng, L.; Tian, F.; Xu, X.-Y.; Wang, L.-X. Tetrahedron 2014, 70, 8665.

(13) Quintard, A.; Rodriguez, J. Adv. Synth. Catal. 2016, 358, 3362.

(14) Urbanietz, G.; Atodiresei, I.; Enders, D. Synthesis 2014, 46, 1261.

(15) Maity, R.; Gharui, C.; Sil, A. K.; Pan, S. C. Org. Lett. 2017, 19, 662.

(16) Bania, N.; Pan, S. C. Org. Biomol. Chem. 2019, 17, 1718.

(17) Mondal, K.; Pan, S. C. J. Org. Chem. 2018, 83, 5301.

(18) Gharui, C.; Behera, D.; Pan, S. C. Adv. Synth. Catal. 2018, 360 , 4502.

(19) (a) Bertelsen, S.; Jørgensen, K. A. Chem. Soc. Rev. 2009, 38, 2178. (b) Melchiorre, P.; Marigo, M.; Carlone, A.; Bartoli, G. Angew. Chem. Int. Ed. 2008, 47, 6138.

(20) (a) Quintard, A.; Constantieux, T.; Rodriguez, J. Angew. Chem. Int. Ed. 2013, 52, 12883. (b) Roudier, M.; Constantieux, T.; Quintard, A.; Rodriguez, J. Org. Lett. 2014, 16, 2802. (c) Roudier, M.; Constantieux, T.; Rodriguez, J.; Quintard, A. Chimia 2016, 70, 97.

(21) For reviews, see: (a) Quintard, A.; Rodriguez, J. Angew. Chem. Int. Ed. 2014, 53, 4044. (b) Quintard, A.; Rodriguez, J. ChemSusChem 2016, 9, 28. (c) For a selected example of the use of this complex, see: Casey, C. P.; Guan, H. J. Am. Chem. Soc. 2007, 129, 5816.

(22) (a) Roudier, M.; Constantieux, T.; Quintard, A.; Rodriguez, J. ACS Catal. 2016, 6, 5236. (b) Rodriguez, J.; Quintard, A. Chimia 2018, $72,580$.

(23) For the limited reactivity of ketoesters in the fragmentation, see: Quintard, A.; Roudier, M.; Rodriguez, J. Synthesis 2018, 50, 785.

(24) Zhu, Y.; Zhang, L.; Luo, S. J. Am. Chem. Soc. 2016, 138, 3978.

(25) Maity, R.; Pan, S. C. Org. Biomol. Chem. 2018, 16, 1598.

(26) Qian, J.; Yi, W.; Huang, X.; Jasinski, J. P.; Zhang, W. Adv. Synth. Catal. 2016, 358, 2811.

(27) (a) Grenning, A. J.; Tunge, J. A. Angew. Chem. Int. Ed. 2011, 50, 1688. (b) Grenning, A. J.; Tunge, J. A. J. Am. Chem. Soc. 2011, 133, 14785. For more recent examples, see: (c) Jha, M.; Chou, T.-Y.; Blunt, B. Tetrahedron 2011, 67, 982. (d) Maji, T.; Ramakumar, K.; Tunge, J. A. Chem. Commun. 2014, 50, 14045. (e) Kumar, N.; Das, M. K.; Ghosh, S.; Bisai, A. Chem. Commun. 2017, 53, 2170. (f) Zhou, X.-L.; Ren, L.; Wang, P.-S. J. Org. Chem. 2017, 82, 9794. (g) Ortega-Martínez, A.; de Lorenzo, R.; Sansano, J. M.; Nájera, C. Tetrahedron 2018, 74, 253.

(28) Grenning, A. J.; Van Allen, C. K.; Maji, T.; Lang, S. B.; Tunge, J. A. J. Org. Chem. 2013, 78, 7281. 
(29) Mukherjee, H.; McDougal, N. T.; Virgil, S. C.; Stoltz, B. M. Org. Lett. 2011, 13, 825.

(30) (a) Beck, T. M.; Breit, B. Angew. Chem. Int. Ed. 2017, 56, 1903. (b) For the racemic version, see: Beck, T. M.; Breit, B. Org. Lett. 2016, 18, 124.

(31) For pioneering examples of the synthesis of medium-sized rings by ring expansion through Claisen-type fragmentation, see: (a) Mahajan, J. R. Synthesis 1976, 110. (b) Mahajan, J. R.; Carvalho, H. D. Synthesis 1979, 518. (c) Mahajan, J. R.; Resck, I. S. Synthesis 1980, 998. (d) Bhat, V.; Cookson, R. C. J. Chem. Soc., Chem. Commun. 1981, 1123. (e) Cookson, R. C.; Ray, P. S. Tetrahedron Lett. 1982, 23, 3521. (f) Kostova, K.; Lorenzi-Riatsch, A.; Nakashita, Y.; Hesse, M. Helv. Chim. Acta 1982, 65, 249. (g) Onu,
N.; Miyake, H.; Kaji, A. J. Org. Chem. 1984, 49, 4997. (h) Schore, N. E.; Nadji, S. D. J. Org. Chem. 1987, 52, 5296. (i) Xie, Z.-F.; Suemune, H.; Sakai, K. J. Chem. Soc., Chem. Commun. 1988, 612. (j) Giorgi, G.; Miranda, S.; López-Alavarado, P.; Avendano, C.; Rodriguez, J.; Menéndez, J. C. Org. Lett. 2005, 7, 2197. (k) Coquerel, Y.; Bensa, D.; Doutheau, A.; Rodriguez, J. Org. Lett. 2006, 8, 4819. (l) Coquerel, Y.; Filippini, M.-H.; Bensa, D.; Rodriguez, J. Chem. Eur. J. 2008, 14, 3078.

(32) Roudier, M.; Constantieux, T.; Quintard, A.; Rodriguez, J. Eur. J. Org. Chem. 2015, 5709.

(33) Zhou, Y.; Wei, Y-L.; Rodriguez, J.; Coquerel, Y. Angew. Chem. Int. Ed. 2019, 58, 456. 